\title{
Associação entre amamentação, fatores obstétricos e perinatais com o desenvolvimento neuropsicomotor infantil
}

Association between breastfeeding, obstetric and perinatal factors with the child neuropsychomotor development

Asociación entre lactancia materna, factores obstétricos y perinatales con el desarrollo de neuropsicomotores infantiles

Recebido: 16/05/2021 | Revisado: 24/05/2021 | Aceito: 30/05/2021 | Publicado: 13/06/2021

Manuela Carla de Souza Lima Daltro

ORCID: https://orcid.org/0000-0003-4034-2411 Centro Universitário de Patos, Brasil

E-mail: manueladaltro@ fiponline.edu.br

Regina Oliveira Bezerra

ORCID: https://orcid.org/0000-0002-4576-9644

Centro Universitário de Patos, Brasil

E-mail: reginaabelo17@gmail.com

Bruna Alencar Castro

ORCID: https://orcid.org/0000-0002-1794-8856 Centro Universitário de Patos, Brasil

E-mail: alencar@hotmail.com

André Luiz Dantas Bezerra

ORCID: https://orcid.org/0000-0002-0547-5772

Centro Universitário de Patos, Brasil

Faculdade São Francisco da Paraíba, Brasil

E-mail: milenanunes@fiponline.edu.br

Michelline Nunes Alves de Sousa

ORCID: https://orcid.org/0000-0002-2729-4921

Prefeitura Municipal de São José do Egito, Brasil

E-mail: michellinesousa@gmail.com

Larissa de Araújo Batista Suárez

ORCID: https://orcid.org/0000-0002-6658-5019

Universidade Católica de Pernambuco, Brasil

Faculdade São Francisco da Paraíba, Brasil

E-mail: labsuarez@gmail.com

Vandezita Dantas de Medeiros Mazzaro

ORCID: https://orcid.org/0000-0002-7297-6032 Centro Universitário de Patos, Brasil E-mail: vanmazzaro@hotmail.com

Miguel Aguila Toledo

ORCID: https://orcid.org/0000-0001-6882-8814 Centro Universitário de Patos, Brasil

E-mail: migueltoledo@fiponline.edu.br

Alanna Michely Batista de Morais ORCID: https://orcid.org/0000-0002-4441-9714 Centro Universitário de Patos, Brasil

E-mail: alannamorais@fiponline.edu.br

Milena Nunes Alves de Sousa

ORCID: https://orcid.org/0000-0001-8327-9147 Centro Universitário de Patos, Brasil

Faculdade São Francisco da Paraíba, Brasil

E-mail: milenanunes@fiponline.edu.br

\section{Resumo}

Objetivou-se avaliar a associação rentre amamentação, fatores obstétricos e perinatais com o desenvolvimento neuropsicomotor infantil. O estudo tratou de uma pesquisa de campo, descritiva, exploratória e quantitativa, que teve como objetivo analisar a associação entre amamentação, fatores obstétricos e perinatais com o desenvolvimento neuropscicomotor (DNPM) infantil. A amostra foi formada por 52 mães que tiveram filhos com idade de 6 até 12 meses de idade e que estavam realizando procedimentos na unidade básica de saúde do município de Patos, Paraíba. A coleta de dados se deu com aplicação de um questionário semiestruturado, em que se avaliou o DNPM, dados do parto, fatores obstétricos, dados da amamentação e associação com os dados do DNPM. Os resultados mostram que a maioria das crianças que fizeram parte da amostra possui DNPM compatível com a idade, quanto mais tempo 
amamentada mais em conformidade com o desenvolvimento adequado. O estudo também mostrou que mães que tiveram traumas durante a gestação e crianças que nasceram abaixo da idade gestacional possuíam DNPM não compatível com a idade. Foi possível constatar os benefícios trazidos pela prática de amamentação exclusiva e sua atuação positiva no desenvolvimento infantil, bem como foi possível discutir os fatores gestacionais que estão associados no DNPM dessas crianças.

Palavras-chave: Aleitamento materno; Desenvolvimento infantil; Criança.

\begin{abstract}
The objective of this study was to evaluate the association between breastfeeding, obstetric and perinal factors with child neuropsychomotor development. The study was a descriptive, exploratory and quantitative field research, which aimed to analyze the association between breastfeeding, obstetric and perinatal factors with child neuropsychomotor development (DPNP). The sample consisted of 52 mothers who had children aged 6 to 12 months old and who were undergoing procedures at the basic health unit, in the city of Patos, Paraiba. Data collection was applied with the application of a semi-structured questionnaire, in which the DPNP, delivery data, obstetric factors, breastfeeding data and association with the DPNP data were evaluated. The results show that the majority of the children who were part of the sample have dnpm compatible with age, the longer breastfed the more in accordance with the appropriate development. The study also showed that mothers who had trauma during pregnancy and children born below gestational age had DPNP not compatible with age. It was possible to verify the benefits brought by the practice of exclusive breastfeeding and its positive performance in child development, as well as it was possible to discuss the gestational factors that are associated in the DPNP of these children.
\end{abstract}

Keywords: Breastfeeding; Child development; Child.

\title{
Resumen
}

El objetivo de este estudio era evaluar la asociación entre la lactancia materna, los factores obstétricos y perinales con el desarrollo neuropsicómotor infantil. El estudio fue una investigación de campo descriptiva, exploratoria y cuantitativa, que tuvo como objetivo analizar la asociación entre la lactancia materna, factores obstétricos y perinatales con el desarrollo neuropsíquico y motor (DNPM) infantil. La muestra estuvo conformada por 52 madres que tenían hijos de 6 a 12 meses de edad y que estaban siendo intervenidos en la unidad básica de salud, de la ciudad de Patos, Paraíba. La recopilación de datos se aplicó con la aplicación de un cuestionario semiestructurado, en el que se evaluaron los datos de entrega, los factores obstétricos, los datos de lactancia materna y la asociación con los datos de la DNPM. Los resultados muestran que la mayoría de los niños que formaron parte de la muestra tienen DNPM compatible con la edad, cuanto más tiempo amamantado más de acuerdo con el desarrollo adecuado. El estudio también mostró que las madres que tuvieron traumas durante el embarazo y los niños nacidos por debajo de la edad gestacional no tenían DNPM compatible con la edad. Fue posible verificar los beneficios que aporta la práctica de la lactancia materna exclusiva y su desempeño positivo en el desarrollo infantil, así como la posibilidad de discutir los factores gestacionales que se asocian en la DNPM de estos niños.

Palabras clave: Lactancia materna; Desarrollo infantil; Niño.

\section{Introdução}

As práticas alimentares na infância são de extrema importância e influencia no estado nutricional das crianças, sendo necessários sua avaliação e seu monitoramento (Saldan, Venancio, Saldiva, Vieira, \& Mello, 2017). A amamentação tem bastante importância para o desenvolvimento da criança, o leite materno é um alimento rico em nutrientes que são fundamentais para o crescimento saudável da criança. É importante que nos dois primeiros trimestres a criança seja alimentada apenas com o leite materno, estudos mostram que crianças amamentadas exclusivamente possuem um menor risco de sobrepeso e são melhores nutridas (Carvalho et al., 2017).

O aleitamento materno exclusivo ocorre quando a criança é amamentada até o sexto mês de vida sem nenhum tipo de introdução ou fórmula alimentar, nesse tempo é indicado que a criança seja só amamentada, pois, o leite materno supre toda a necessidade da criança, o AME ainda é desconhecido por grande parte das nutrizes brasileiras, principalmente as de baixo poder aquisitivo (Oliveira, Silva, \& Silva, 2018).

Os benefícios dessa prática devem ser explicados às mulheres, desde o pré- natal, até o pós-parto. Cabe aos profissionais de saúde clarificar para essas gestantes suas dúvidas e explicar os benefícios trazidos pelo leite materno tanto pra saúde da mãe como a do bebê (Coutinho; Soares; \& Fernandes, 2014). Caminha et al. (2014) afirmam que a amamentação é a estratégia que mais previne morbimortalidades infantil, além de ser extremamente importante para o desenvolvimento físico e 
mental nos primeiros anos de vida, impedindo que doenças se manifestem na vida adulta, como por exemplo morbidades crônicas.

Severiano et al. (2017) observaram diferença expressiva entre os grupos que fizeram aleitamento materno exclusivo (AME) por 6 meses e os que fizeram por menos de 6 meses, constatando-se que crianças amamentadas exclusivamente por menos de 6 meses apresentaram mais chances no atraso do desenvolvimento neuropsicomotor (DNPM), quando comparadas as que tiveram 6 meses de AME. Muitos são os fatores obstétricos que influenciam o DNPM da criança, desde fatores pré-natais, fatores nutricionais, distúrbios hormonais, pré-termo de gestação e entre outros (Pinto, Muller, \& Medeiros, 2019).

Quanto às complicações causadas pela infecção urinária não tratada na gestação, deixa o RN mais vulnerável a ter um atraso no seu desenvolvimento (Duarte, Marcolin, Quintana, \& Cavalli, 2008). Asseveram com a afirmativa outros autores, ao evidenciaram que a infecção urinária materna grave, durante a gestação, é forte responsável pelo risco de atraso no desenvolvimento em crianças até um ano de idade (Ribeiro, Perosa, \& Padovani, 2014).

Diante do exposto, objetivou-se avaliar a associação entre amamentação, fatores obstétricos e perinatais com o desenvolvimento neuropsicomotor infantil.

\section{Metodologia}

Pesquisa de campo, descritiva, exploratória e quantitativa que foi realizada no município de Patos, estado da Paraíba. A população utilizada na pesquisa foi formada por todas as mães de crianças de 6 até 12 meses que estiveram fazendo algum procedimento na Unidade Básica de Saúde (UBS) Maria Marques e foi possível pesquisar com 96,3\% (n=52) do universo de pesquisa ( $\mathrm{n}=52$ ). A amostra do tipo não probabilística por conveniência foi adotada, conforme o estabelecimento dos critérios de inclusão: ter filho de 6 a 12 meses cadastrado na UBS citada no período de setembro e outubro de 2020, período de realização da coleta de dados; e de exclusão: ser menor de 18 anos e mães com baixa capacidade de responder o instrumento por questões psíquicas. Teve-se uma perda de duas $(3,7 \%)$ mães, pois uma era menor de idade e a outra apresentava distúrbio psicológico.

A coleta de dados foi feita a partir do questionário aplicado presencialmente. As questões contemplaram amamentação exclusiva, idade da criança, fatores sociais, demográficos perinatais e obstétricos relacionados à gravidez. Os dados da amostra foram analisados e tabulados utilizando o Statistical Package for the Social Sciences (SSPSS, versão 23.0).

Para classificar o DNPM foi realizado um raciocínio matemático, em que se calcularam os valores para pontuar os parâmetros de desenvolvimento das crianças avaliadas no estudo: 1) 0 a 8 - não compatível com a idade (a criança não atingia nem 30\% das habilidades psicomotoras); 2) 9 a 16 - parcialmente compatível com a idade (as crianças ficavam entre $30 \%$ a $60 \%$ das habilidades para idade); e 3) 17 a 25 - totalmente compatível com a idade (mais de 60\% das habilidades psicomotoras). Tais parâmetros atenderam o preconizado pela Sociedade Brasileira de Pediatra (SBP, 2017). Além de estatística básica (frequência relativa e absoluta), utilizou-se o teste de correlação de Pearson, adotando-se nível de significância de $\mathrm{p} \leq 0,05$.

A realização deste estudo considerou a Resolução nº 510/16 e 580/18 do Conselho Nacional de Saúde que rege sobre a ética da pesquisa envolvendo seres humanos direta ou indiretamente, assegurando a garantia de que a privacidade do sujeito da pesquisa fora preservada. Foi aprovado pelo Comitê de Ética em Pesquisa com Seres Humanos do Centro Universitário de Patos, CAAE: 26327819.2.0000.5181, parecer de número 3.909.584/2020. 


\section{Resultados}

A média de idades das mães estudadas foi de 28,16, com idade mínima de 18 e máxima de 42 anos de idade, desvio padrão de 6,7. Verificou-se que 40,3\% das mães estudadas eram do lar. Quanto a variável de escolaridade, 40,3\% da amostra concluiu o ensino médio, $42,2 \%$ possuíam estrutura familiar e 63,5\% dessas mães tinham renda mensal de até R\$ 1000,00 .

Quanto à das crianças estudadas, em que a maior parte obteve um desenvolvimento totalmente compatível com porcentagem de 40,4\%, porém $21,2 \%$ alcançaram DNMP incompatível coma idade.

Tabela 1. Descrição das características das lactantes pesquisadas, na cidade de Patos-PB.

\begin{tabular}{|c|c|c|}
\hline VARIÁVEL & $\mathbf{N}$ & $\%$ \\
\hline OCUPAÇÃO & 2 & 3,8 \\
\hline Do lar & 21 & 40,3 \\
\hline Autônoma & 12 & 30 \\
\hline Estudante & 1 & 1,7 \\
\hline Desempregada & 6 & 11 \\
\hline Profissionais atuantes em sua área de formação & 7 & 17 \\
\hline \multicolumn{3}{|l|}{ ESCOLARIDADE } \\
\hline Ensino fundamental incompleto & 16 & 30,8 \\
\hline Ensino médio completo & 22 & 42,3 \\
\hline Ensino Médio incompleto & 6 & 11,5 \\
\hline Ensino superior completo & 2 & 3,8 \\
\hline Ensino superior incompleto & 3 & 5,9 \\
\hline Não Alfabetizada & 1 & 1,9 \\
\hline \multicolumn{3}{|l|}{ RAÇA } \\
\hline Branca & 18 & 34,6 \\
\hline Parda & 13 & 25 \\
\hline Mestiça & 12 & 23,1 \\
\hline Negra & 9 & 17,3 \\
\hline \multicolumn{3}{|l|}{ ESTADO CIVIL } \\
\hline Solteira & 12 & 23,1 \\
\hline Casada & 23 & 44,2 \\
\hline União Estável & 16 & 30,8 \\
\hline Divorciada & 1 & 1,9 \\
\hline \multicolumn{3}{|l|}{ RENDA } \\
\hline Acima de $\mathrm{R} \$ 1.000,00$ & 16 & 30,8 \\
\hline Até R\$1.000,00 & 33 & 63,5 \\
\hline Não Especificou & 3 & 5,7 \\
\hline \multicolumn{3}{|l|}{ CLASSIFICAÇÃO DO DNPM } \\
\hline 0 a 8 - Incompatível com a idade. & 11 & 21,2 \\
\hline 9 a 16 - Parcialmente compatível com a idade. & 20 & 38,4 \\
\hline 17 a 25 - Totalmente compatível com a idade. & 21 & 40,4 \\
\hline
\end{tabular}

Fonte: Autores.

A Tabela 2 descreve a relação das características obstétricas e perinatais das lactantes e crianças pesquisadas associadas com o DNPM. Mostraram-se resultados relevantes, uma vez que mães que tiveram traumas na gestação, apenas $35,7 \%(\mathrm{p}=0,02 * *)$ das crianças não apresentam DNPM compatível; mães que fizeram uso de cigarro na gestação apresentaram $50 \%$ dos resultados para DNPM incompatível $\left(\mathrm{p}=0,02^{* *}\right)$ e $50 \%$ parcialmente compatível ( $\left.\mathrm{p}=0,06^{* *}\right)$.

Outra característica obstétrica expressiva é a idade gestacional pré-termo em que $70 \%(\mathrm{p}=0,00 * *)$ a qual se associou com o DNPM, evidenciando-se incompatível com a idade. 
Tabela 2. Descrição das características obstétricas das lactantes pesquisadas, na cidade de Patos-PB e sua relação com o DNPM.

\begin{tabular}{|c|c|c|c|c|c|}
\hline $\begin{array}{l}\text { FATORES } \\
\text { OBSTÉTRICOS }\end{array}$ & $\begin{array}{c}\text { DNPM incompatível } \\
(0-8)\end{array}$ & $\begin{array}{c}\text { DNPM parcialmente } \\
\text { compatível } \\
(9-16) \\
\end{array}$ & $\begin{array}{c}\text { DNPM totalmente } \\
\text { compatível } \\
(17-25) \\
\end{array}$ & $\begin{array}{c}\text { Total } \\
(\%)\end{array}$ & $P^{*}$ \\
\hline $\begin{array}{l}\text { Gravidez na } \\
\text { adolescência }\end{array}$ & & & & & 0,26 \\
\hline SIM & 100 & $0,00 \%$ & $0.0 \%$ & $100 \%$ & \\
\hline NÃO & 0.0 & $0.0 \%$ & $10 \%$ & $10 \%$ & \\
\hline Traumas na gestação & & & & & $\mathbf{0 , 0 2} * *$ \\
\hline SIM & $35,70 \%$ & $7.2 \%$ & $57.1 \%$ & $100 \%$ & \\
\hline NÃO & $15.8 \%$ & $50.0 \%$ & $34.2 \%$ & $100 \%$ & \\
\hline $\begin{array}{l}\text { Doenças adquiridas na } \\
\text { gravidez }\end{array}$ & & & & & 0,17 \\
\hline Diabetes Gestacional & $100 \%$ & $0,00 \%$ & $0.0 \%$ & $100 \%$ & \\
\hline Hipertensão & $100 \%$ & $0,00 \%$ & $0,00 \%$ & $100 \%$ & \\
\hline Eclampsia & $0,00 \%$ & $42,90 \%$ & $57,10 \%$ & $100 \%$ & \\
\hline Depressão Pós-parto & $50,00 \%$ & $50,00 \%$ & $0,00 \%$ & $100 \%$ & \\
\hline $\begin{array}{l}\text { Incontinência Urinária } \\
\text { pós-parto }\end{array}$ & $15,40 \%$ & $34,60 \%$ & $50,00 \%$ & $100 \%$ & \\
\hline $\begin{array}{l}\text { Nenhuma Doença } \\
\text { adquirida }\end{array}$ & 36,6 & 35,5 & 27,9 & $-100 \%$ & \\
\hline $\begin{array}{l}\text { Infeção Urinária pós- } \\
\text { parto }\end{array}$ & $0,00 \%$ & $-66,80 \%$ & $-33,20 \%$ & $-100 \%$ & \\
\hline HÁBITOS DE VIDA & $\begin{array}{c}\text { DNPM incompatível } \\
(0-8)\end{array}$ & $\begin{array}{c}\text { DNPM parcialmente } \\
\text { compatível } \\
(9-16) \\
\end{array}$ & $\begin{array}{c}\text { DNPM totalmente } \\
\text { compatível } \\
(17-25) \\
\end{array}$ & $\begin{array}{c}\text { Total } \\
(\%)\end{array}$ & $P^{*}$ \\
\hline $\begin{array}{l}\text { Uso de cigarro na } \\
\text { gestação }\end{array}$ & & & & & $\mathbf{0 , 0 2} * *$ \\
\hline $\operatorname{Sim}$ & $50 \%$ & $50 \%$ & $0 \%$ & $100 \%$ & \\
\hline Não & $15.9 \%$ & $36.4 \%$ & $47,70 \%$ & $100 \%$ & \\
\hline $\begin{array}{l}\text { Uso de bebidas } \\
\text { alcoólicas na gestação }\end{array}$ & & & & & $0,06 *$ \\
\hline Sim & $18.2 \%$ & $36.4 \%$ & $45.4 \%$ & $100 \%$ & \\
\hline Não & $46.3 \%$ & $39.0 \%$ & $14.7 \%$ & $100 \%$ & \\
\hline $\begin{array}{l}\text { PÓS-PARTO } \\
\text { FATORES } \\
\text { PERINATAIS } \\
\end{array}$ & $\begin{array}{c}\text { DNPM incompatível } \\
(0-8)\end{array}$ & $\begin{array}{c}\text { DNPM parcialmente } \\
\text { compatível } \\
(9-16)\end{array}$ & $\begin{array}{c}\text { DNPM totalmente } \\
\text { compatível } \\
(17-25)\end{array}$ & $\begin{array}{c}\text { Total } \\
(\%)\end{array}$ & $P^{*}$ \\
\hline Idade Gestacional & & & & & $0,00 * *$ \\
\hline \multirow{3}{*}{ Pré-Termo } & $7(70 \%)$ & $2(20 \%)$ & $1(10 \%)$ & $10(10 \%)$ & \\
\hline & $70 \%$ & $20,00 \%$ & $10,00 \%$ & $100 \%$ & \\
\hline & $2(20 \%)$ & $4(40 \%)$ & $3(30 \%)$ & $9(90 \%)$ & \\
\hline Pós-termo & $22,20 \%$ & $\begin{array}{c}44,40 \% \\
0\end{array}$ & $33,30 \%$ & $100 \%$ & \\
\hline \multirow[t]{2}{*}{ Termo } & $2(20 \%)$ & $14(14 \%)$ & $17(17 \%)$ & $33(33 \%)$ & \\
\hline & $6,10 \%$ & $42,40 \%$ & $51,50 \%$ & $100 \%$ & \\
\hline
\end{tabular}

*Correlação de Pearson;

** Estatisticamente significativa.

Fonte: Autores.

A Tabela 3 não mostrou valores estatisticamente significativos $(\mathrm{p}=0,28)$, porém seus valores evidenciaram que quanto mais tempo essa criança foi amamentada mais ela possui o DNPM compatível com a sua idade. 
Tabela 3. Descrição do tempo de aleitamento materno exclusivo das lactantes pesquisadas, na cidade de Patos-PB e sua relação com o DNPM de seus filhos.

\begin{tabular}{|c|c|c|c|c|c|}
\hline $\begin{array}{l}\text { IDADE EM QUE FICOU EM } \\
\text { AME }\end{array}$ & $\begin{array}{c}\text { DNPM } \\
\text { incompatível } \\
(0-8)\end{array}$ & $\begin{array}{c}\text { DNPM parcialmente } \\
\text { compatível } \\
(9-16)\end{array}$ & $\begin{array}{l}\text { DNPM totalmente } \\
\text { compatível } \\
(17-25)\end{array}$ & Total $(\%)$ & $P^{*}$ \\
\hline Não mamou & $3(50 \%)$ & $1(16,7 \%)$ & $2(50 \%)$ & $6(100 \%)$ & \\
\hline $1^{\circ}$ mês & $3(60 \%)$ & $2(40 \%)$ & $0(0,0 \%)$ & $5(100 \%)$ & \\
\hline $2^{\circ}$ meses & $1(16,7 \%)$ & $3(50 \%)$ & $2(33,3 \%)$ & $6(100 \%)$ & \\
\hline $3^{\circ}$ meses & $2(20 \%)$ & $5(50 \%)$ & $3(30 \%)$ & $10(100 \%)$ & 0,28 \\
\hline $4^{\circ}$ meses & $0(0,0 \%)$ & $1(25,0 \%)$ & $3(75,0 \%)$ & $4(100,0 \%)$ & \\
\hline $5^{\circ}$ meses & $0(0,0 \%)$ & $0(0,0 \%)$ & $0(0,0 \%)$ & $0(0,0 \%)$ & \\
\hline $6^{\circ}$ meses & $1(16,7 \%)$ & $5(33,3 \%)$ & $9(60,0 \%)$ & $15(100,0 \%)$ & \\
\hline AINDA ESTÁ EM AME & $1(16,7 \%)$ & $3(50 \%)$ & $2(33,3 \%)$ & $6(100 \%)$ & \\
\hline TOTAL & $11(100 \%)$ & $20(100 \%)$ & $22(100 \%)$ & $52(100 \%)$ & \\
\hline
\end{tabular}

Fonte: Autores.

\section{Discussão}

O estudo mostrou que a maioria das mães amamentaram exclusivamente seus filhos e isso pode estar relacionado aos dados de escolaridade, estado civil e renda. Em relação à escolaridade, observou-se que 43,3\% das mães possuíam ensino médio completo e esses dados exibiram que as mesmas apresentam um bom nível de escolaridade, considerando adequando para o entendimento e compreensão conveniente de informações para amamentação exclusiva.

Queluz, Pereira, Santos, Leite e Ricco (2012) e Dias, Freitas, Martins, Martins e Alves (2016) evidenciaram que o desmame precoce tem relação com escolaridade inadequada, isso mostrou que a escolaridade é um fator preditivo para a prática de AME, podendo trazer diversos prejuízos e mantendo essa prática de forma menos consciente.

Santos et al. (2019) afirmaram que a escolaridade mais elevada é um fator que influencia positivamente a prática de AME. Os autores ao observarem mulheres que possuíam um nível mais alto de escolaridade, amamentaram seus filhos por mais tempo, pois tiveram mais acesso a informações sobre a importância da AME. Neste estudo em Patos, as mães possuíam ensino médio completo, um preditor relevante, pois a maioria das crianças estudadas não foi desmamada precocemente.

Ademais, a partir de uma revisão integrativa, foi possível elencar fatores que influenciam o desmame precoce do aleitamento materno, destacando-se: baixo nível de escolaridade das mães, a mãe trabalhar fora de casa, traumas mamilares, leite fraco, deficiência na consulta de pré-natal e uso de bicos artificiais (chupeta e mamadeira) (Pinheiro, Nascimento, \& Vetorazo, 2021). De modo análogo, outra revisão apontou como principais motivos à descontinuidade da amamentação exclusiva antes dos seis meses de vida os fatores culturais, socioeconômicos, retorno da genitora ao trabalho, intercorrências mamárias, uso de chupetas, baixa escolaridade e ausência de orientação (Araújo, Souza, Bomfim, \& Santos, 2021).

Outra informação importante foi assinalada por Rocci e Fernandes (2014), os quais asseguraram que a presença de um companheiro fixo contribui para que as mães prolonguem o seu período de lactação. O estudo de Lima, Cazola e Pícoli (2017) também enfatizou o estado civil casado como um bom preditor para a continuidade da amamentação.

A grande maioria, equivalente a 57,8\% recebiam abaixo de R\$1000,00 por mês, eram de baixa renda e provavelmente essas mães não possuem renda própria e são sustentas pelos pais das crianças ou pela família. Mesmo com baixa renda e a ausência de emprego (43,3\% das mães), talvez tenha servido como benéfico a AME, pois o trabalho aparece como um fator que interfere na efetividade do aleitamento materno (Ribeiro, Florentino, Mariano, Peres, \& Rodrigues, 2017). As mulheres que exerciam atividades remuneradas fora do lar precisavam desempenhar sua jornada de trabalho longe de suas casas e, por esse motivo, acaba interferindo no processo de aleitamento materno. 
O estudo de Tavares et al. (2020) mostrou significância em seus resultados onde mães que possuem baixa renda têm mais chances de permanecer e alcançar o tempo de AME adequado, devido a prática alimentar ser uma forma mais econômica e sustentável de alimentar uma criança pequena.

Por conseguinte, foi possível observar que 40,4\% das crianças estudadas apresentaram DNPM compatível a sua idade, porém possui uma amostra significativa de $21,2 \%$ que não estavam dentro do padrão motor relacionado à idade. $\mathrm{E}$ algumas mães relataram traumas sofridos durante a gestação que foram acidentes domésticos, infecções urinárias durante a gestação, traumas psicológicos, descolamento de placenta, hemorragia, fizeram uso de bebidas alcoólicas e cigarro, possivelmente esses fatores interferiram no desenvolver das crianças estudadas.

A infecção no trato urinário (ITU) materna durante a gestação é um dos traumas responsáveis pelo risco de atraso no desenvolvimento em crianças (Ribeiro, Perosa, \& Padovani, 2014), mesmo que as mães tenham realizado acompanhamento pré-natal no período gestacional. Furtado, Mendonça, Lameira e Ferreira (2018) analisaram a infecção urinária como um fator de risco para atraso no desenvolvimento motor, em que $40 \%$ das mães estudadas tiveram ITU durante a gravidez. Pode-se observar que quase metade da população estudada teve essa infecção no período gestacional, um fator de risco preocupante para atraso no DNPM.

Também, Gliorsi, et al. (2020) evidenciaram em seu estudo de series de casos, que as mães participantes da pesquisa possuíam alguns fatores perinatais como hipertensão, maturação placentária precoce, sangramentos, pontos esses que são indícios para prematuridade que gera uma preocupação para um possível atraso motor da criança posteriormente. Importante lembrar que muitos são influenciam o DNPM da criança, sejam pré-natais, nutricionais, hormonais, entre outros (Pinto, Muller, \& Medeiros, 2019).

O estudo revelou, adicionalmente, que 50\% apresentaram o tabagismo durante a gravidez com variáveis incompatíveis (compatível e parcialmente compatível) com o desenvolvimento motor infantil. O tabagismo materno durante a gravidez e a amamentação pode afetar todas as fases do desenvolver da vida da criança, ocasionando assim graves consequências, pois toda a vida do indivíduo pode gerar programações cruciais do seu desenvolvimento motor (Massago, \& Dworak, 2018).

Maia, Pereira e Menezes (2015) afirmaram que o tabagismo pode ocasionar problemas perinatais como os citados pelas mães no estudo, como: episódios de hemorragia na gravidez, descolamento de placenta e nascimentos prematuros, devido à nicotina isso pode ocasionar falta de oxigenação pra placenta.

A prematuridade é considerada um fator de risco para o desenvolvimento neuropsicomotor, como apresentado neste trabalho. Estudos já mostram a existência de uma ligação entre o DNPM e a prematuridade (Righi et al., 2017; Israel, Pileggi, Krambeck, \& Piveta, 2020; Lodi, Trubian, Sangali, Rodrigues, \& Saccani, 2020; Monteiro et al., 2020).

Em relação à amamentação exclusiva, não foi possível observar estatisticamente valores significativos, porém o trabalho mostrou que quanto mais tempo à criança foi amamentada mais atendeu padrões compatíveis com desenvolvimento para a idade. Estudo com crianças prematuras mostrou que o AME até os 6 meses de idade se tornou bastante eficiente na evolução do DNPM infantil, por isso a importância de não amamentar com fórmulas alimentares antes dos 6 meses de idade (Severiano et al., 2017).

Miatello et al. (2019) encontraram que o tempo de AME foi um fator positivo para o DNPM adequado com a idade. Tudehope, Fewtrell, Kashyap e Udaeta (2013) verificaram resultados significativos, apontando efeitos positivos da AME associada com DNPM infantil. Ainda, estudos apontaram que crianças amamentadas obtiveram habilidades motoras mais precocemente quando comparadas aquelas não alimentadas com leite da mãe (Sabando, 2006; Andres et al., 2012; Bulla, 2021; Castro, Suárez, Vieites, Bergadá, \& Cassinelli, 2021). Embora as evidências indiquem associação entre a amamentação e o DNPM, alguns achados mostram-se controversos aos resultados (Eickmann et al., 2007; Guxens et al., 2011). 
Por conseguinte, destacam-se com mecanismos e fatores que podem ser índices para clarificar essa associação, a influência dos ácidos graxos poli-insaturados de cadeia longa, em especial, o ácido araquidônico (AA) e o docosahexaenóico (DHA), subtende-se que são os principais responsáveis para uma melhor desenvoltura adquirida por crianças amamentadas (Gibson \& Makrides, 2001; Reynolds, 2001; Hallowell, \& Spatz, 2012; Belfort, 2017). Estudos sugerem que estes índices procedem na biogênese das membranas celulares, na neurotransmissão e no acolhimento contra os estresses oxidativos (Gartner et al., 2005; Shaikh, \& Chantry, 2006; Eidelman, 2012; Updegrove, 2013).

Keim et al. (2012), em seu estudo mostraram crianças que tiveram a prática de AME nos quatro primeiros meses, apresentaram maiores benefícios quanto ao DNPM aos 12 meses comparadas com crianças desmamadas precocemente e amamentados com fórmulas. No entanto, após observar ajustes para nascimento pré-termo, sexo, raça/etnia e educação, a prática alimentar exclusiva não houve indicativo de desenvolvimento nas crianças estudadas.

\section{Conclusão}

A amamentação exclusiva é uma estratégia ideal para promoção de saúde, cabe aos profissionais de saúde explanar e simplificar essa prática para as gestantes, desmistificando crenças e fazendo um acompanhamento das mesmas durante o pósnatal, assim a criança vai ter um crescimento saudável.

A partir dos achados desta pesquisa foi possível constatar que o aleitamento materno exclusivo associou-se com DNPMI e também com fatores obstétricos e perinatais. Foi evidenciado que as crianças que ficaram em AME até a idade adequada estavam dentro dos padrões de desenvolvimento motor ideal para sua idade.

Embora não seja possível afirmar assertivamente a correlação hábitos de vida e desfechos motores na infância há indícios que alguns hábitos de vida durante a gestação podem estar associados ao atraso no desenvolvimento motor do grupo. Portanto, sugere-se que estudos prospectivos do tipo coorte sejam desenvolvidos.

\section{Referências}

Andres, A.; Cleves, M. A.; Bellando, J. B.; Pivik, R. T.; Casey, P. H. \& Badger, T. M. (2012). Developmental status of 1-year-old infants fed breast milk, cow's milk formula, or soy formula. Pediatrics, 129(6), 1134-1140.

Araújo, S. C.; Souza, A. D. A.; Bomfim, A. N. A. \& Santos, J. B. (2021). Fatores intervenientes do desmame precoce durante o aleitamento materno exclusivo. Revista Eletrônica Acervo Saúde, 13(4), e6882-e6882.

Belfort, M. B. (2017). The science of breastfeeding and brain development. Breastfeeding Medicine, 12(8), 459-461.

Bulla, F. B. (2021). El hierro en la alimentación complementaria del niño lactante: una revisión. Perspectivas en Nutrición Humana, 23(1).

Caminha, M. D. F. C.; Azevedo, P. T. Á. C. C. D.; Sampaio, B. B.; Acioly, V. M. C. D.; Belo, M. P. M.; Lira, P. I. C. D. \& Batista Filho, M. (2014). Aleitamento materno em crianças de 0 a 59 meses no Estado de Pernambuco, Brasil, segundo o peso ao nascer. Ciência \& Saúde Coletiva, 19, $2021-2032$.

Carvalho, J. L. D. S.; Cirino, I. P.; Lima, L. H. D. O.; Sousa, A. F. D.; Carvalho, M. F. D. \& Oliveira, E. A. R. (2016). Conhecimento das mães sobre aleitamento materno exclusivo e alimentação complementar. Saúde Redes, 383-392.

Castro, S.; Suárez, C. V.; Vieites, A.; Bergadá, I, \& Cassinelli, H. (2021). Raquitismo vinculado al uso de fórmulas elementales: Reporte de caso. Arch. argent. pediatr, e49-e53.

Coutinho, A. C. F. P.; Soares, A. C. O. \& Fernandes, P. S. (2014). Conhecimento das mães sobre os benefícios do aleitamento materno à saúde da mulher. Revista Enfermagem UFPE online, 8 (5), 1213-1220.

Dias, E. G.; Freitas, A. L. S. A.; Martins, H. C. S. C.; Martins, K. P. \& Alves, J. C. S. (2016). Vantagens da amamentação e alterações no estilo de vida da lactante. Revista Contexto \& Saúde, 16(31), 25-33. Recuperado de https://revistas.unijui.edu.br/index.php/contextoesaude/article/view/5763

Duarte, G.; Marcolin, A. C.; Quintana, S. M. \& Cavalli, R. C. (2008). Infecção urinária na gravidez. Revista Brasileira de Ginecologia e Obstetrícia, 30(2), 93-100.

Eickmann, S. H.; Lira, P. I. C.; Lima, M. D. C.; Coutinho, S. B.; Teixeira, M. D. L. P. D. \& Ashworth, A. (2007). Breast feeding and mental and motor development at 12 months in a low-income population in northeast Brazil. Paediatric and perinatal epidemiology, 21(2), 129-137.

Eidelman, A. I. (2012). Breastfeeding and the use of human milk: an analysis of the American Academy of Pediatrics 2012 Breastfeeding Policy 
Statement. Breastfeeding medicine, 7(5), 323-324.

Furtado, M. A. S.; Mendonça, A. S. G. B.; Lameira, A. B. C. \& Ferreira, L. F. (2018). Avaliação do desenvolvimento motor de lactentes dependentes de servidores em instituição de ensino superior no Amazonas. Saúde e Desenvolvimento Humano, 6(1), 29-38. Recuperado de http://dx.doi.org/10.18316/sdh.v6i1.4096

Gartner, L. M.; Morton, J.; Lawrence, R. A.; Naylor, A. J.; O'Hare, D.; Schanler, R. J. \& Eidelman, A. I. (2005). Breastfeeding and the use of human milk. Pediatrics, 115(2), 496-506.

Gibson, R. A. \& Makrides, M. (2001). Long-chain polyunsaturated fatty acids in breast milk. Bioactive components of human milk, 375-383.

Gliorsi, M. J.; Salerno, G. R. F.; Callegari, M. R.; Fernandes, M. F.; Tropiano, L. M. D. C. C. \& de Assis, S. M. B. (2020). Desenvolvimento de lactentes prétermos-Série de casos. Brazilian Journal of Health Review,3(3), 6407-6423. Recuperado https://www.brazilianjournals.com/index.php/BJHR/article/view/11666

Guxens, M.; Mendez, M. A.; Moltó-Puigmartí, C.; Julvez, J.; García-Esteban, R.; Forns, J., ... \& Sunyer, J. (2011). Breastfeeding, long-chain polyunsaturated fatty acids in colostrum, and infant mental development. Pediatrics, 128(4), e880-e889.

Hallowell, S. G. \& Spatz, D. L. (2012). The relationship of brain development and breastfeeding in the late-preterm infant. Journal of Pediatric Nursing, 27(2), 154-162.

Israel, M. A. R. D.; Pileggi, Y.; Krambeck, T. V. \& Piveta, F. C. P. (2020). Intervenção precoce no desenvolvimento neuromotor de lactentes prematuros de risco: Early intervention in the neuromotor devolopment of premature infants at risk. Revista FisiSenectus, 8(1), 1-18. Recuperado de https://bell.unochapeco.edu.br/revistas/index.php/fisisenectus/article/view/5171

Keim, S. A.; Daniels, J. L.; Siega-Riz, A. M.; Herring, A. H.; Dole, N. \& Scheidt, P. C. (2012). Breastfeeding and long-chain polyunsaturated fatty acid intake in the first 4 post-natal months and infant cognitive development: an observational study. Maternal \& child nutrition, 8(4), 471-482.

Lima, J. P.; Cazola, L. H. O. \& Pícoli, R. P. (2017). A participação do pai no processo de amamentação. Cogitare Enfermagem, 22(1). Recuperado de https://revistas.ufpr.br/cogitare/article/view/47846

Lodi, M. N.; Trubian, F.; Sangali, C. C, Rodrigues, L. M. \& Saccani, R. (2020). A Influência das Práticas Maternas no Desenvolvimento Motor de Crianças Prematuras. A Influência das Práticas Maternas no Desenvolvimento Motor de Crianças Prematuras. Revista Brasileira de Ciências da Saúde, 24 (3), 505-514.

Maia, J. A.; Pereira, L. A. \& Menezes, F. A. (2016). Consequências do uso de drogas durante a gravidez. Revista Enfermagem Contemporânea, 4(2). http://dx.doi.org/10.17267/2317-3378rec.v4i2.664

Massago, M. \& Dworak, E. S. (2018). Efeitos transgeracionais do tabagismo materno durante a gestação e amamentação. Infarma-Ciências Farmacêticas, 30(1), 44-49. Recuperado de http://dx.doi.org/10.14450/2318-9312.v30.e1.a2018.pp44-49

Miatello, I.; Pellarin, L. A.; Nascimento, M. I. S.; Boque, M. R.; Galbetti, V. H. \& Elias, L. S. D. T. (2019). Seguimento ambulatorial dos recém-nascidos de alto risco de um hospital-escola do noroeste paulista. CuidArte, Enferm, 106-110.

Monteiro, P. V. O.; Cardoso, A. B. R.; Costa, L. L.; Caldas, I. F. R.; Cunha, K. C. \& Chermont, A. G. (2020). Associações entre desenvolvimento motor e sociocomunicativo de prematuros e interação mãe-bebê. Revista Brasileira de Educação e Saúde, 10(3), 177-183. Recuperado de https://www.editoraverde.org/gvaa.com.br/revista/index.php/REBES/article/view/7976

Oliveira, T. C.; Silva, M. D. M. G. \& Silva, J. B. (2018). Revisão sobre a importância do aleitamento materno exclusivo nos primeiros seis meses de vida para a dupla mãe-bebê. Revista de Iniciação Científica e Extensão, 1(Esp 2), 250-254.

Pinheiro, B. M.; Nascimento, R. C. \& Vetorazo, J. V. P. (2021). Fatores que influenciam o desmame precoce do aleitamento materno: uma revisão integrativa. Revista Eletrônica Acervo Enfermagem, 11, e7227-e7227.

Pinto, S. S.; Muller, J. E. \& Medeiros, C. A. A. (2019). Causas de origem obstétrica ou materna relacionadas ao atraso no desenvolvimento neuropsicomotor de crianças avaliadas pela escala CAT/CLAMS. Arquivos Catarinenses de Medicina, 48(3), 02-13.

Queluz, M. C.; Pereira, M. J. B.; Santos, C. B. D.; Leite, A. M. \& Ricco, R. G. (2012). Prevalence and determinants of exclusive breastfeeding in the city of Serrana, São Paulo, Brazil. Revista da Escola de Enfermagem da USP, 46(3), 537-543.

Reynolds, A. (2001). Breastfeeding and brain development. Pediatric Clinics of North America, 48(1), 159-171.

Ribeiro, D. G.; Perosa, G. B. \& Padovani, F. H. P. (2014). Fatores de risco para o desenvolvimento de crianças atendidas em Unidades de Saúde da Família, ao final do primeiro ano de vida. Ciência \& Saúde Coletiva, 19, 215-226.

Ribeiro, K. V.; Florentino, C. L. V.; Mariano, D. C. A.; Peres, P. L. P. \& Rodrigues, B. M. R. D. (2017). A amamentação e o trabalho informal: a vivência de mães trabalhadoras. Revista Pró-UniverSUS, 8(2), 03-09. Recuperado de http://editora.universidadedevassouras.edu.br/index.php/RPU/article/view/504

Righi, N. C.; Martins, F. K.; Hermes, L.; Rosa, K. M.; Böck, T. H. O. \& Trevisan, C. M. (2017). Influência da correção da idade na detecção de riscos no desenvolvimento motor de prematuros. Saúde e Pesquisa, 10(3), 417-421. Recuperado de http://dx.doi.org/10.177651/1983-1870.2017v10n3p417-421

Rocci, E. \& Fernandes, R. A. Q. (2014). Dificuldades no aleitamento materno e influência no desmame precoce. Revista Brasileira de Enfermagem, 67(1), 2227.

Sabando, M. E. M. (2006). Lactancia materna vs. alimentación con fórmulas lácteas. Estudio comparativo de sus efectos en el desarrollo psicomotriz en niños entre 6 a 24 meses de edad, realizado en el hospital Guayaquil “Abel Gilbert Pontón”, octubre 2004-marzo 2005. Medicina, 11(1), 25-32. 
Research, Society and Development, v. 10, n. 7, e5210716152, 2021

(CC BY 4.0) | ISSN 2525-3409 | DOI: http://dx.doi.org/10.33448/rsd-v10i7.16152

Saldan, P. C.; Venancio, S. I; Saldiva, S. R. D. M.; Vieira, D. G. \& Mello, D. F. D. (2017). Consumo de leites em menores de um ano de idade e variáveis associadas ao consumo de leite não materno. Revista Paulista de Pediatria, 35(4), 407-414.

Santos, E. M.; Silva, L. S.; Rodrigues, B. F. S.; Amorim, T. M. A. X.; Silva, C. S.; Borba, J. M. C. \&Tavares, F. C. L. P;. (2019). Avaliação do aleitamento materno em crianças até dois anos assistidas na atenção básica do Recife, Pernambuco, Brasil. Ciência \& Saúde Coletiva, 24(3), 1211-1222.Recuperado de https://doi.org/10.1590/1413-81232018243.126120171

Sociedade Brasileira de Pediatria (2017). Tratado de Pediatria (4a ed.). Barueri, SP: Manole.

Tavares, A. M. C.; Silva, F. R.; Callou, M. A. M.; Silva, R. F.; Morais, M. P. S. \& Rocha, É. M. B. (2020). Fatores que interferem na duração do aleitamento materno de crianças na Região Metropolitana do Cariri cearense. DEMETRA: Alimentação, Nutrição \& Saúde, 15, 47367. Recuperado de https://doi.org/10.12957/demetra.2020.47367

Severiano, A. A. D. O.; Dantas, D. D. S.; Oliveira, V. L. C. D.; Lopes, J. M.; Souza, D. E. D. \& Magalhães, A. G. (2017). Association between breastfeeding, obstetric factors and child development in northeast Brazil. Journal of Human Growth and Development, 27(2), 158-165.

Shaikh, U. \& Chantry, C. (2006). Reflections on the American Academy of Pediatrics' 2005 policy statement on "Breastfeeding and the use of human milk". Journal of human lactation, 22(1), 108-110.

Tudehope, D.; Fewtrell, M.; Kashyap, S. \& Udaeta, E. (2013). Nutritional needs of the micropreterm infant. The Journal of pediatrics, 162(3), S72-S80.

Updegrove, K. H. (2013). Donor human milk banking: growth, challenges, and the role of HMBANA. Breastfeeding Medicine, 8(5), $435-437$. 